\title{
Modulation of narrowband and broadband gamma connectivity in retinal degeneration mice according to electrical stimulation pulse width
}

\author{
Stephen Kugbere Agadagba ${ }^{1, *}$ and Leanne Lai Hang Chan ${ }^{1,2}$ \\ ${ }^{1}$ Department of Electrical Engineering, City University of Hong Kong, Hong Kong, People's Republic of China \\ ${ }^{2}$ Centre for Biosystems, Neuroscience, and Nanotechnology, City University of Hong Kong, Hong Kong, People's Republic of China
}

\begin{abstract}
Brain connectivity involves the structural, functional and effective communication between neurons across brain regions and is expressed in neuronal oscillations. Previous research has reported the evidence of two types of gamma oscillations namely the broadband gamma $(30 \mathrm{~Hz}-90 \mathrm{~Hz})$ and narrowband gamma $(55 \mathrm{~Hz}-70 \mathrm{~Hz})$ oscillations which have been implicated in excitatory and inhibitory network transmission. There is presently no systematic investigation of the relationship between electrical stimulation pulse width and narrow or broadband gamma oscillations in visual-deficient mice. In the current study, we set out to bridge this gap in knowledge by exploring the modulation of brain connectivity indices in broadband gamma and narrowband gamma oscillations in response to varying electrical stimulation pulse width in retinal degeneration (rd) mice. The results revealed that a low pulse width $(0.5 \mathrm{~ms} / \mathrm{phase})$ strongly enhances coherence and directional connectivity of broadband and narrowband gamma oscillations in contra visual cortex and contra prefrontal cortex of rd mice. This study serves a crucial role in the design and utilisation of visual prostheses by contributing to the understanding of information transmission between different brain regions under retinal electrical stimulation in visual-deficit population.
\end{abstract}

KEYWORDS Biomedical engineering; retinal degeneration; electrical stimulation; connectivity; retinal prosthesis CONTACT Stephen Kugbere Agadagba@ sagadagba3-c@my.cityu.edu.hk

Received 17 March 2020

\section{Introduction}

The brain contains a huge network of interconnected neural pathways which interact in a synchronised manner through electrical activities along nerve fibre tracts. These synchronised activities can be readily detected and studied by electroencephalography (EEG) and electrocorticography (ECoG), then imaged using brain connectivity analyses. These types of analyses are specifically used to represent neural communication networks required for normal brain functionality (Haufe et al., 2018; Zhang et al., 2015). For several decades, development in imaging techniques for brain networks have shown that closer and more distant brain regions are linked and able to communicate with each other (Cabral et al., 2014; Chou et al., 2015; Mancuso et al., 2019). Moreover, research has shown that neurodegenerative disorders are capable of disrupting brain functions and brain connectivity patterns (Cauda et al., 2018; Iturria-Medina and Evans, 2015). Remediation and treatments have also been demonstrated to change brain functions. In essence, studying the pattern and directionality of brain connectivity in neurodegenerative diseases, particularly retinal degeneration which involves loss of neural input to the visual cortex, is of paramount importance in the development of possible therapies for restoration of the normal physiological state.

Brain networks have a vast neural population that acts in uniformity in order to transmit signals from one brain region to another. This interaction could be of functional or causal origin. Functional connectivity reveals sites of the brain with similar oscillations or frequencies of corresponding activity. Such brain regions may play key roles in the resting (task-free) state or processing of higher order (task-related) signals that are necessary for motor responses, sensory responses and cognitive processing (Towle et al., 2007). Causal or effective connectivity makes use of functional connectivity information and additionally estimates the influence one neural signal may have over another neural signal (Shovon et al., 2016). Specifically, while functional connectivity is directionless, effective connectivity determines the directional flow of neural information between brain sites. Functional and effective connectivity estimates can be analysed by techniques like coherence and transfer entropy, respectively, in the frequency domain. Coherence is a common mathematical approach for determining frequency-dependent correlations and estimating the degree of similarity in the activity of the brain recorded at divergent brain sites (Borjigin et al., 2013). Transfer entropy on the other hand is a method to mathematically compute the directionality of neuronal information flow across brain sites (Saba et al., 2019) i.e. which direction of the brain is the neural information sent to and which direction is the information received or relayed. This implies that transfer entropy could be either in the feedforward (FF) or feedback (FB) direction of information transfer.

Gamma oscillations are low amplitude rhythms that are produced by a wide variety of neural circuits. These fast oscillations have been hypothesised to represent multiple 
phenomena within the cortical and subcortical regions of the brain (Headley and Paré, 2017). Scientific investigations have demonstrated the evidence of two distinct types of gamma oscillations, namely the broadband gamma and narrowband gamma oscillations. The former rhythm has broader spectral peaks and oscillates in a frequency band between $30 \mathrm{~Hz}$ and $90 \mathrm{~Hz}$. The latter rhythm varies less in frequency, thus has narrower spectral peaks and oscillates at a frequency band between $55 \mathrm{~Hz}$ and $70 \mathrm{~Hz}$. Both types of the aforementioned oscillations have been reportedly linked to vital roles of inhibitory and excitatory neural networks, respectively (Saleem et al., 2017; Storchi et al., 2017).

To the best of our knowledge, scientific literature contains no systematic investigation of the relationship, if any, between electrical stimulation pulse width and narrow or broadband gamma oscillations in retinal degeneration mice. Here we address this gap in knowledge and demonstrate that brain connectivity in both broad and narrowband gamma oscillations are strongly modulated by electrical stimulation pulse width and that the increased connectivity at low pulse width is both functionally and causally correlated. Therefore, this study serves as a crucial step towards using the reported data as a template to produce more effective strategies for stimulation, particularly in the design and utilisation of visual prostheses.

\section{Materials and methods}

\subsection{Animals used and surgery steps}

To mimic retinal degeneration in humans, $\mathrm{rd} 10$ mice were used as model animals of the human blinding disease called retinitis pigmentosa. These mice were bred and housed in City University of Hong Kong Laboratory Animal Research Unit (CityU LARU). All experiments described in this study were conducted by the following methods endorsed by Animal Research Ethics Subcommittee in City University of Hong Kong (CityU) and Health Department, HKSAR. For the current study, male and female mice (total $n=40$ ) aged P60 - P90 were used. Surgical craniotomy was done in a similar way as in the previous work (Agadagba et al., 2019). In brief, mice were first anesthetised by injection of ketamine-xylazine (Ketamine: $100 \mathrm{mg} / \mathrm{kg}$; Xylazine: $10 \mathrm{mg} / \mathrm{kg}$ ). Subsequently, oxygen-mixed isoflurane anesthesia (O2: $0.4 \%$, Iso: $1.5 \%$ ) was administered to maintain the sleep-state during the surgical procedure. Mice were head-fixed on the stereotaxis with dura exposed and four stainless steel bone-screw electrodes (shaft Ø: $2.4 \mathrm{~mm}$ ) (Decorah LLC, GEC, North America) were implanted on the dura surface. The bonescrew electrode served as the recording electrode and specifically implantation was done in a bilateral direction on the visual cortex (coordinates: anterior-posterior: -3.5 $\mathrm{mm}, 2.5 \mathrm{~mm}$ from the midline of the skull) and prefrontal cortices (coordinates: anterior-posterior: $2 \mathrm{~mm}, 1 \mathrm{~mm}$ from the midline of the skull). A reference bone-screw was positioned in the cerebellar region. During the entire surgery, regular application of lubritha (Alfamedics, Hong Kong, HKSAR) was done to keep the mice's eyes moisturised. Also, mice heart beats were closely monitored and the body temperature was kept constant at $38^{\circ} \mathrm{C}$ with a heat pad (Model \# TP702; Gaymar Industries, Inc., NY, USA).

\subsection{Electrical stimulation protocol}

For electrical stimulation, the procedure was similar to the previous work (Agadagba et al., 2019). Mice's eyes were electrically stimulated transcorneally. Firstly, mice were anesthetised with oxygen-mixed isoflurane anesthesia (O2: $0.4 \%$, Iso: $1.5 \%$ ) and a silver stimulating electrode (diameter: $0.4 \mathrm{~mm}$, impedance: $89 \mathrm{k} \Omega$ at $100 \mathrm{kHz}$ ) placed on the right cornea of mice (surface area: $7.07 \mathrm{~mm}^{2}$ ) was connected to an electrical pulse generator (STG4004, Multi-Channel Systems, Baden-Württemberg, Germany). The reference electrode was inserted subcutaneously near the stimulated eye. Transcorneal electrical stimulation (TES) was done for a prolonged duration (30 minutes/day) and was repeated for an additional four days. There were four study groups consisting of three experimental groups [stimulated with a constant current of $400 \mu \mathrm{A}$ at $10 \mathrm{~Hz}$ frequency and varying pulse width of $0.5 \mathrm{~ms} /$ phase (charge injected: $0.2 \mu \mathrm{C}$ ), $2 \mathrm{~ms} /$ phase (charge injected: $0.8 \mu \mathrm{C}$ ) and $5 \mathrm{~ms} /$ phase (charge injected: $2 \mu \mathrm{C}$ ) biphasic pulses, respectively] and one sham group (no stimulation current was applied).

\subsection{Resting-state ECoG recording}

A-M Systems 3600 (A-M Systems, Washington, US) amplifier and CED Micro 1401-3 (Cambridge Electronic Design, UK) were used to acquire the resting-state ECoG signals from the implanted electrodes in awake rd10 mice. A transfer cable was used to connect the implanted electrodes and the aforementioned data acquisition systems. The resting-state ECoG was recorded in a room with normal lighting. The recorded signals were sampled at $5 \mathrm{kHz}$ by filtering from $0.3 \mathrm{~Hz}$ to $100 \mathrm{~Hz}$. The base-line data (pre-stimulation) was recorded for three days (for 10 minutes/day). Following the prolonged TES, resting-state ECoG activity (post-stimulation) was recorded (for 10 minutes/day) for three days.

\subsection{Brain connectivity detection}

After data acquisition, $50 \mathrm{~Hz}$ notch filter from MATLAB, R2018b (MathWorks Inc.) algorithm was applied to the data to filter out the interfering $50 \mathrm{~Hz}$ noise and its possible super-harmonics originating from the alternating current (AC) line. Broadband gamma $(30 \mathrm{~Hz}$ 
- $90 \mathrm{~Hz})$ and narrowband gamma $(55 \mathrm{~Hz}-70 \mathrm{~Hz})$ were filtered respectively using MATLAB, R2018b (MathWorks Inc.) algorithm. Undirected and directed brain connectivity between the left visual cortex (contra Vcx) and left prefrontal cortex (contra PFx) were analysed by coherence and normalised symbolic transfer entropy (NSTE), respectively.

\subsubsection{Analysis of coherence}

Coherence was analysed on the basis of power spectrum estimation. The coherence between contra Vcx and contra PFx was computed as magnitude squared coherence $C_{x y}(f)$ using the "mscohere. $m$ " function in MATLAB, R2018b signal processing toolbox (MathWorks Inc.). The value computed gives an estimate of the coherence between the input signals $x$ and $y$ based on the modified periodogram technique by Welch. $C_{x y}(f)$ is a realvalued frequency-dependent function that ranges between 0 and 1 indicating the extent of correlation between $x$ and $y$ per frequency.

$$
C_{x y}(f)=\frac{\left|P_{x y}(f)\right|^{2}}{P_{x x}(f) P_{y y}(f)}, 0 \leq C_{x y}(f) \leq 1 .
$$

In the above equation, $P_{x x}(f)$ and $P_{y y}(f)$ are represented as the power spectral density (PSD) of the input signal $x$ and signal $y$, respectively. $P_{x y}(f)$ represents the cross-PSD. In the present study, the mean coherence between the contra Vcx and contra PFx was calculated for all mice in the awake state. During the entire recording, ECoG signals were split into 2 seconds epochs having 1 second overlap. Then, $C_{x y}(f)$ was calculated at each frequency bin and epoch in the broadband gamma and narrowband gamma frequency ranges respectively.

\subsubsection{Analysis of Normalised Symbolic Transfer Entropy}

FF and FB directional connectivity between the contra Vcx and left contra PFx in broadband and narrowband gamma oscillations were calculated respectively by Normalised Symbolic Transfer Entropy (NSTE). NSTE is the Symbolic Transfer Entropy (STE) normalised by elimination of the possible STE bias through subsequent surrogate data analysis. It is important to note that NSTE estimates the causal influence of a source signal $X$ on a target signal $Y$.

$$
\operatorname{NSTE}_{X \rightarrow Y}=\frac{\text { STE }_{X \rightarrow Y^{-}} \text {STE }_{X \rightarrow Y}^{\text {Shuffled }}}{H\left(Y^{F} \mid Y^{P}\right)} \in[0,1] .
$$

In Equation (2), I $\left[Y^{F} ; X^{P}, Y^{P}\right]$ and I $\left[Y^{F} ; Y^{F}\right]$ denote mutual information values. $X^{F}, Y^{F}$, and $Y^{F}$ are the past of source and target signals, and the future of the target signal, respectively. The difference between these two mutual information values computes the transfer of information from signal $X$ to signal $Y$. In the current study, EEGlab V15 (Swartz Center for Computational Neuroscience, CA) and EEGapp (BIAPT lab, McGill University) toolboxes were used in conjunction with MATLAB, R2018b (MathWorks Inc.) to calculate the NSTE.

\subsection{Statistical analysis}

Comparison between connectivity in sham and stimulated groups was done by one-way analysis of variance (ANOVA). All graphical plots, representations and statistical analysis were made using Origin(Pro) (Version 2015b). Unpaired (two-sample) student's t-test was used to obtain the significant difference $(p<0.05)$ in connectivity between each stimulated group and sham group. All graphs were represented with error bars which denote \pm standard error of the mean (SEM).

\section{Results}

\subsection{Effects of pulse width on broadband and narrowband directional connectivity}

In order to determine directional connectivity in broadband and narrowband gamma oscillations of recorded ECoG signals in rd10 mice stimulated at varying pulse width, neural information transfer in contra Vcx and contra PFx was estimated by FF and FB NSTE, respectively. After five days of the prolonged TES, only rd10 mice stimulated with $0.5 \mathrm{~ms} /$ phase pulse width showed significant increase $(\mathrm{p}<0.05)$ in both FF and FB connectivity in broadband gamma and narrowband gamma oscillations, respectively, compared to sham group shown in Figures 1 and 2 (a) 2 (d). Rd10 mice stimulated with other stimulation pulse widths ( $2 \mathrm{~ms} /$ phase and $5 \mathrm{~ms} /$ phase) yielded no significant ( $p>0.05)$ change in FF and FB directional connectivity of broadband gamma and narrowband gamma oscillations shown in Figures 1 and 2 (a) - 2 (d).

\subsection{Effects of pulse width on broadband and narrowband undirected connectivity}

Undirected connectivity in broadband and narrowband gamma oscillations signals of rd10 mice was determined by computing the coherence between contra Vcx and contra PFx brain regions at varying stimulation pulse width with sham stimulated rd10 group. Similar to directed connectivity, the results revealed that after five days of prolonged TES, only rd10 mice stimulated with $0.5 \mathrm{~ms} /$ phase pulse width showed significant increase $(\mathrm{p}<0.05)$ in both broadband and narrowband gamma oscillations coherence, respectively, compared to the sham stimulated group shown in Figures 3 (a) and 3 (b). 
(a)

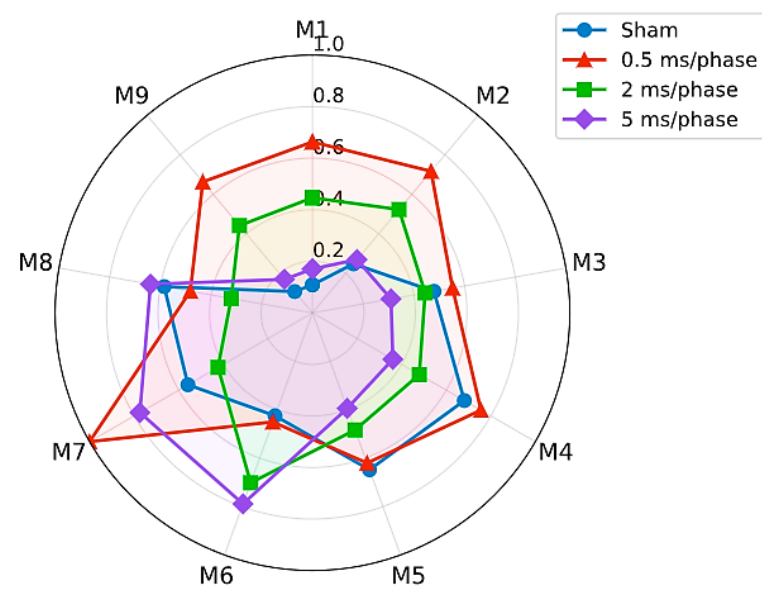

(c)

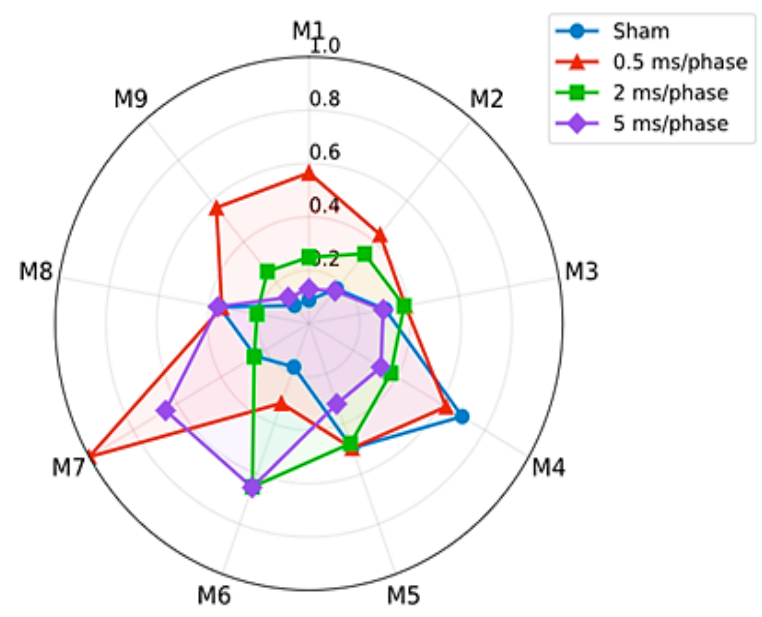

(b)

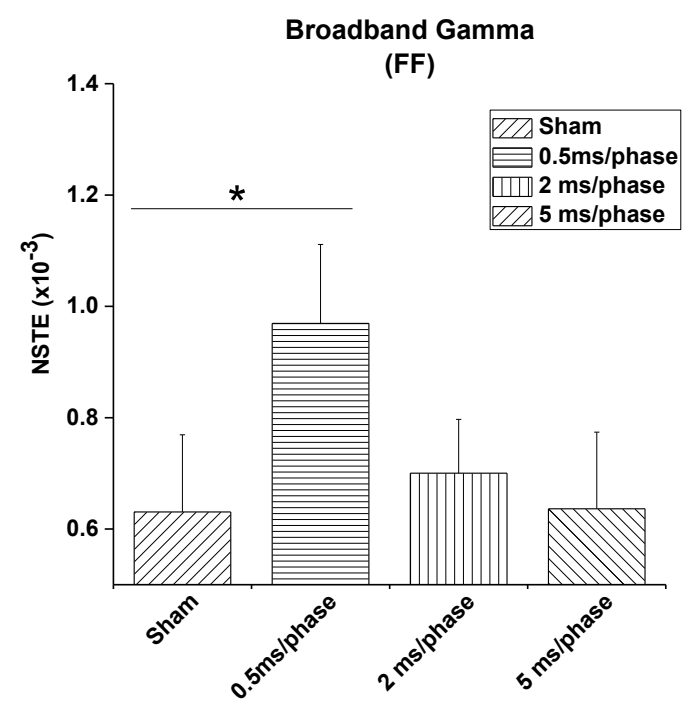

(d)

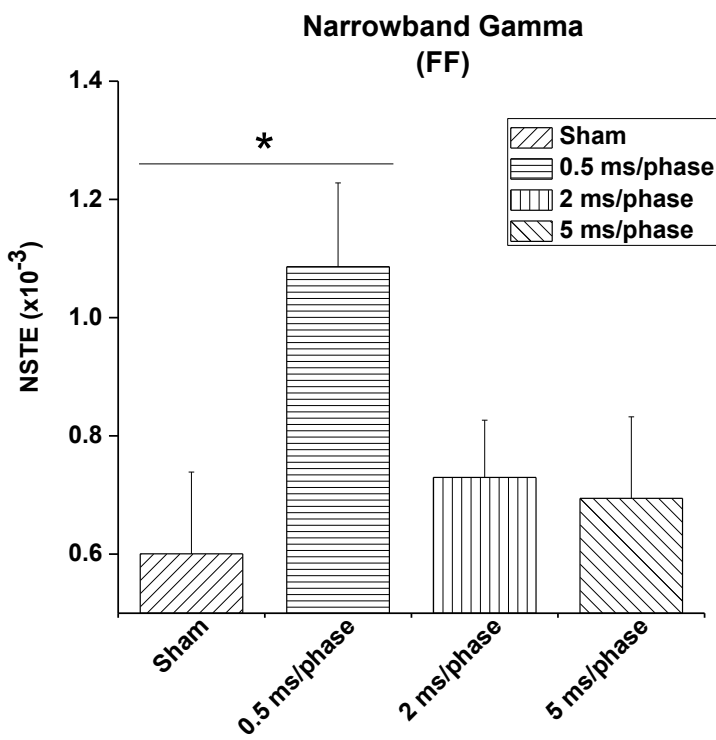

Figure 1. Contra Vcx - contra PFx FF connectivity in rd10 mice after the prolonged TES. NSTE was used to estimate the FF directional connectivity in broadband and narrowband gamma oscillations in contra Vcx - contra PFx of rd10 mice stimulated at varying pulse width of $0.5 \mathrm{~ms} /$ phase, $2 \mathrm{~ms} /$ phase and $5 \mathrm{~ms} /$ phase, respectively. (a) and (c) show normalised values of FF NSTE of broadband and narrowband gamma oscillations, respectively, in contra Vcx - contra PFx of individual rd10 mouse (M). (b) and (d) show the average NSTE $(n=9)$. Only rd10 mice stimulated with 0.5 ms/phase pulse width showed significant increase in contra Vcx - contra PFx FF connectivity of narrowband and broadband gamma oscillations compared to the sham group. *Significant difference $(\mathrm{p}<0.05)$. 
(a)

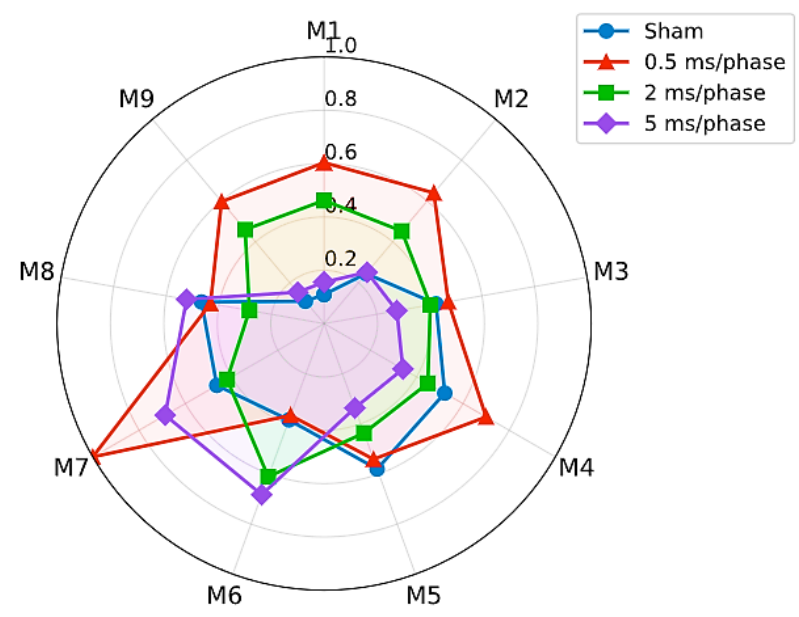

(c)

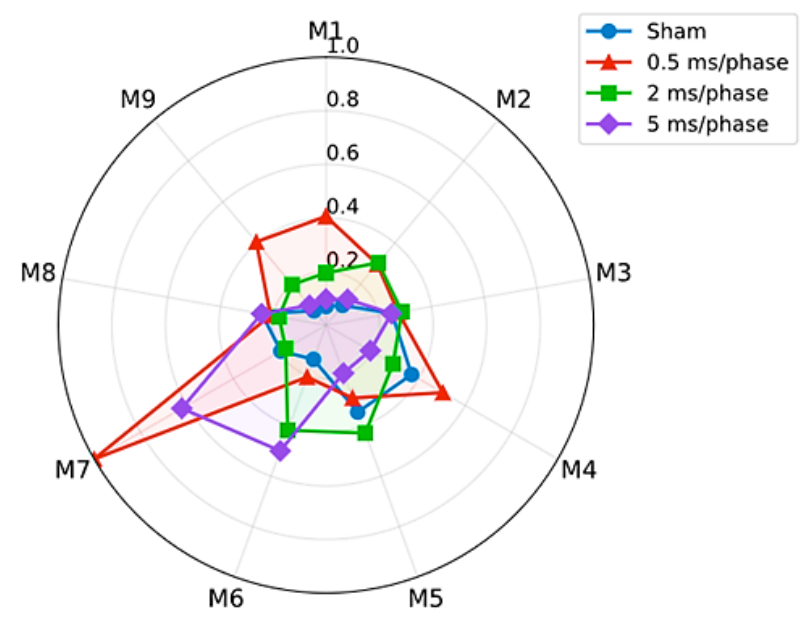

(b)

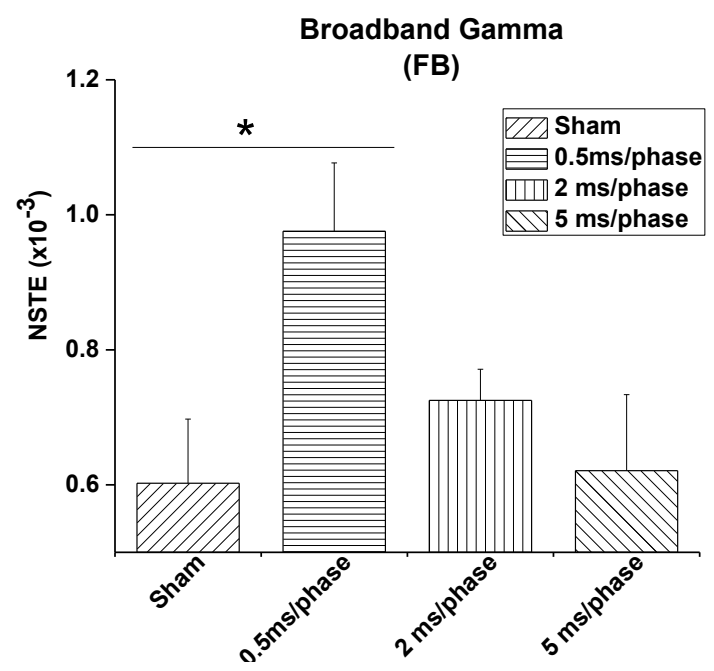

(d)

d) Narrowband Gamma

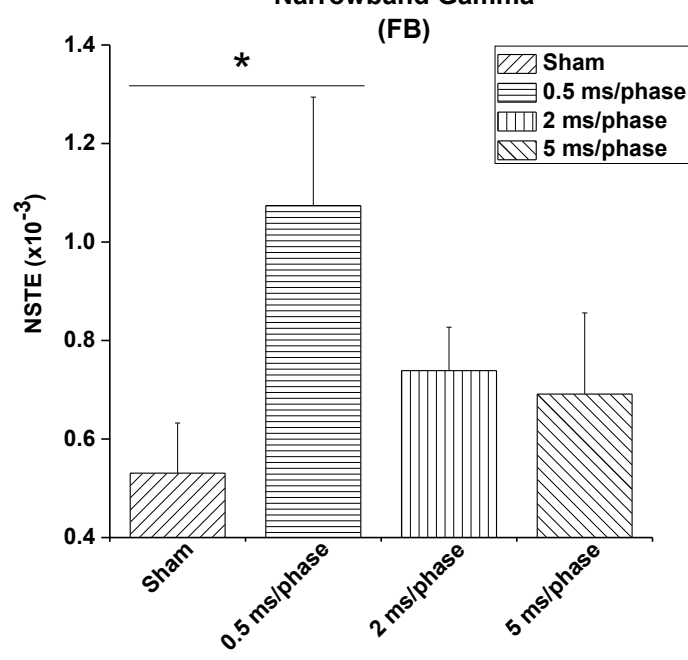

Figure 2. Contra PFx - contra Vcx FB connectivity in rd10 mice after the prolonged TES. NSTE was used to estimate the FB directional connectivity in broadband and narrowband gamma oscillations in contra PFx - contra Vcx of rd10 mice stimulated at varying pulse width of $0.5 \mathrm{~ms} /$ phase, $2 \mathrm{~ms} /$ phase and $5 \mathrm{~ms} /$ phase, respectively. (a) and (c) show normalised values of FB NSTE of broadband and narrowband gamma oscillations, respectively, in contra Vcx - contra PFx of individual rd10 mouse (M). (b) and (d) show the average NSTE $(n=9)$. Only rd10 mice stimulated with 0.5 ms/phase pulse width showed significant increase in contra PFx - contra VCx FB connectivity of narrowband and broadband gamma oscillations compared to the sham group. *Significant difference $(p<0.05)$. 
(a)

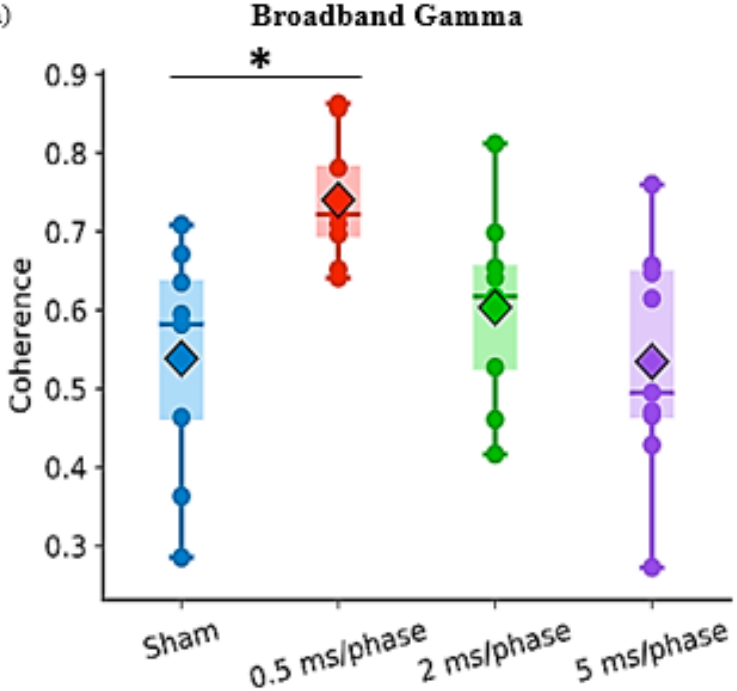

(b)

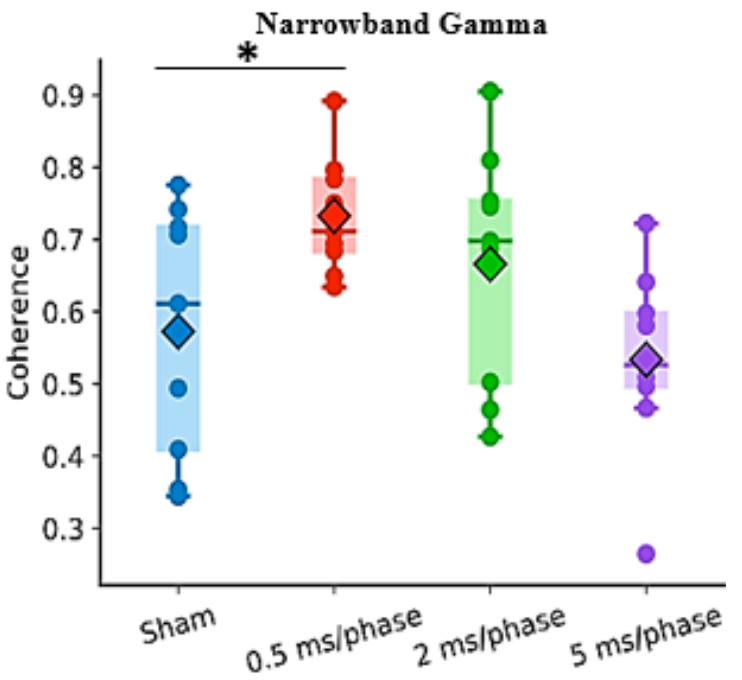

Figure 3. Contra Vcx - contra PFx undirected connectivity in rd10 mice after the prolonged TES. Coherence was used to estimate undirected connectivity in broadband and narrowband gamma oscillations in contra Vcx - contra PFx of rd10 mice stimulated at varying pulse width of $0.5 \mathrm{~ms} /$ phase, $2 \mathrm{~ms} /$ phase and $5 \mathrm{~ms} /$ phase respectively. (a) and (b) are box plots showing the coherence of broadband and narrowband gamma oscillations, respectively, in contra Vcx - contra PFx of individual rd10 mouse. Only rd10 mice stimulated with $0.5 \mathrm{~ms} /$ phase pulse width showed significant increase $(\mathrm{p}<0.05)$ in contra Vcx contra PFx mean coherence compared to the sham group (a) and (b). *Significant difference $(\mathrm{p}<0.05)$.

\section{Discussion}

The present study explored directional and undirected brain connectivity in broadband and narrowband gamma oscillations of electrically stimulated rd10 mice. In directional connectivity analysis, the results demonstrated that a low pulse width $(0.5 \mathrm{~ms} /$ phase $)$ electrical stimulation caused a significant increase in NSTE of both narrowband and broadband gamma oscillations, respectively, compared to the sham stimulated rd10 mice. This increase was observed in both the FF direction (contra Vcx to contra PFx) and FB direction (contra PFx to contra Vcx). Moreover, an increase in directional connectivity was strongly correlated with undirected connectivity as evident by a similar significant increase in coherence of both narrowband and broadband gamma oscillations, respectively, compared to the sham stimulated rd10 mice. This observation suggests that the increase in directed and undirected connectivity occurs via a common pathway or mechanism at a low pulse width electrical stimulation. As previously reported, directed and undirected connectivity are used to detect functional and effective communication respectively across brain regions (Bowyer, 2016). Being that in the current study, NSTE and coherence were correlated at low stimulation pulse width; our results also indicate the increased synchronous activation of neural populations in contra Vcx and contra PFx, after electrical stimulation at low pulse width. Identifying synchronous neural activation is an effective strategy that can be applied in visual prostheses research to detect the efficiency of functional brain connectivity. To this end, using analytical tools of both functional and effective brain connectivity is paramount in visual prostheses community to explore normal and dysfunctional brain neural networks (Goldenberg and Galván, 2015; Hohenfeld et al., 2018; Passamonti et al., 2019) before designing appropriate visual implants. This combined approach of understanding brain network communication in retinal degeneration provides a better and more sustainable multimodal bioindicator than utilising only one type of brain network analysis. Previous researches of broadband gamma oscillations have been reported in several species such as cats, primates, humans and mice (Gray and Singer, 1989; Pesaran et al., 2002; Welle and Contrevas, 2016). Narrowband gamma oscillations have been observed to be prevalent in the visual cortex of free-moving mice in the awake state (Lee et al., 2014; Niell and Stryker, 2010).

Both gamma bands have been reported to have bearings in the retina, LGN and visual cortex where they play key roles in excitation and inhibition network processes. Saleem et al. (2017) investigated both bands and discovered that narrowband gamma oscillations are received from the visual thalamus of mice and increase in power with arousal from the increased light intensity. Broadband and narrowband gamma oscillations have been reported to be generated by the most robust and 
common process for the appearance of gamma rhythm in the neocortex which involves an interaction between inhibitory and excitatory neural networks (Cardin, 2017; Storchi et al., 2016; Welle and Contreras, 2017). Increased narrowband gamma activity has been demonstrated to be mediated by excitatory neural networks while increased broadband gamma activity have been linked to increased inhibitory neural networks (Saleem et al., 2017; Sohal et al., 2009). A balance in excitatory and inhibitory network processes is essential for normal physiological processes (Bhatia et al., 2019) and as aforementioned, several neurological and neurodegenerative disorders are deeply rooted in dysfunctional excitatory-inhibitory brain network connectivity. Given the use of electrical stimulation serves as a therapeutic tool by visual prostheses, this study has potential applications in clinical researches designed to optimise the electrical stimulation modalities used by the visual prostheses community.

\section{Conclusions}

In the current study, we explored the changes in functional and effective brain connectivity of broadband and narrowband oscillations in response to varying pulse width in electrically stimulated retinal degeneration mice. We found that low pulse width is better at increasing brain connectivity in narrowband and broadband oscillations which have previously been described to play key roles in excitation and inhibition of cortical neurons. Clinical research into retinal prostheses will find the data reported here useful by designing prostheses where low pulse width electrical stimulations can be implemented to target increasing brain connectivity in broadband and narrowband oscillations. Moreover, recordings of broadband and narrowband oscillations may offer a novel way to check neural prostheses efficacy for the improvement of stimulation strategies by neural feedback.

\section{Acknowledgments}

We are grateful to Dr Yi Wang, Mr Chung-Yuen Chan and Ms Xin Li for making positive contributions to the experimental setup and protocol of this research. Also, special thanks to Mr Abdelrhaman Eldaly for his assistance towards plotting some of the figures in this study. City University of Hong Kong (under projects 7004831) and Research Grant Council of the Hong Kong Special Administrative Region (CityU 11208218), the Innovation Technology Fund Guangdong-Hong Kong Technology Cooperation Funding Scheme (GHP/078/18GD), and Guangdong Science and Technology Research Program (Grants 2019A050503007) supported this research. Any opinions, findings, conclusions or recommendations expressed in this material/event (or by members of the project team) do not reflect the views of the Government of the HKSAR, the Innovation and Technology Commission or the Innovation and Technology Fund Research Projects Assessment Panel.

\section{Notes on Contributors}

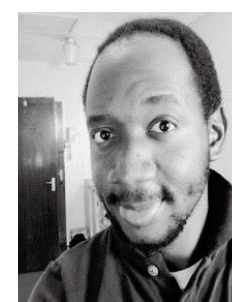

Mr Stephen Kugbere Agadagba received the B.Sc. degree in Biochemistry from the University of Benin, Nigeria in 2008 and the M.Sc. degree in Molecular Biotechnology from the University of Birmingham, United Kingdom in 2012. $\mathrm{He}$ is currently a Ph.D. student in the Department of Electrical Engineering, City University of Hong Kong, Hong Kong. His research interests include ECoG signal analysis, electrical stimulation and in vivo electrophysiology.

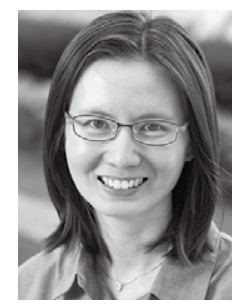

Dr Leanne Lai Hang Chan (M'09SM'19) received the B.Eng. degree in Electrical and Electronic Engineering from The University of Hong Kong, Hong Kong, the M.S. degree in Electrical Engineering and the Ph.D. degree in Biomedical Engineering from the University of Southern California, Los Angeles, California, USA. She has been an Associate Professor in Electrical Engineering with the City University of Hong Kong. Her research interests include retinal prosthesis, implantable electronic system, brain networks, and computer vision.

\section{References}

[1] Agadagba SK, Li X and Chan LL (2019). Electroencephalogram power alterations in retinal degeneration mice after prolonged transcorneal electrical stimulation. In: Proceedings of the 9th International IEEE/EMBS Conference on Neural Engineering (NER). San Francisco: IEEE, pp. 219222.

[2] Bhatia A, Moza S and Bhalla US (2019). Precise excitation-inhibition balance controls gain and timing in the hippocampus. eLife, 8:e43415.

[3] Borjigin J, Lee U, Liu T, Pal D, Huff S, Klarr D, Sloboda J, Hernandez J, Wang MM and Mashour GA (2013). Surge of neurophysiological coherence and connectivity in the dying brain. Proceedings of the National Academy of Sciences of the United States of America, 110 (35), pp. 14432-14437.

[4] Bowyer SM (2016). Coherence a measure of the brain networks: past and present. Neuropsychiatric Electrophysiology, 2 (1). 
[5] Cabral J, Kringelbach ML and Deco G (2014). Exploring the network dynamics underlying brain activity during rest. Progress in Neurobiology, 114, pp. 102-131.

[6] Cardin JA (2016). Snapshots of the brain in action: Local circuit operations through the lens of oscillations. Journal of Neuroscience, 36 (41), pp. 10496-10504.

[7] Cauda F, Nani A, Manuello J, Premi E, Palermo S, Tatu K, Duca S, Fox PT and Costa T (2018). Brain structural alterations are distributed following functional, anatomic and genetic connectivity. Brain, 141 (11), pp. 3211-3232.

[8] Chou WC, Duann JR, She HC, Huang LY and Jung TP (2015). Explore the functional connectivity between brain regions during a chemistry working memory task. Plos One, 10 (6): e0129019.

[9] Goldenberg D and Galván A (2015). The use of functional and effective connectivity techniques to understand the developing brain. Developmental cognitive neuroscience, 12, pp. 155-164.

[10] Gray CM and Singer W (1989). Stimulus-specific neuronal oscillations in orientation columns of cat visual cortex. Proceedings of the National Academy of Sciences of the United States of America, 86 (5), pp. 1698-1702.

[11] Haufe S, Deguzman P, Henin S, Arcaro M, Honey CJ, Hasson U and Parra LC (2018). Reliability and correlation of fMRI, ECoG and EEG during natural stimulus processing. bioRxiv, pp. 1-19.

[12] Headley DB and Paré D (2017). Common oscillatory mechanisms across multiple memory systems. Nature Partner Journals; Science of Learning, 2 (1).

[13] Hohenfeld C, Werner CJ and Reetz K (2018). Resting-state connectivity in neurodegenerative disorders: Is there potential for an imaging biomarker? NeuroImage: Clinical, 18, pp. 849-870.

[14] Iturria-Medina Y and Evans AC (2015). On the central role of brain connectivity in neurodegenerative disease progression. Frontiers in Aging Neuroscience, 7(90), pp. 90-99.

[15] Lee AM, Hoy JL, Bonci A, Wilbrecht L, Stryker MP and Niell CM (2014). Identification of a brainstem circuit regulating visual cortical state in parallel with locomotion. Neuron, 83 (2), pp. 455-466.

[16] Mancuso L, Costa T, Nani A, Manuello J, Liloia D, Gelmini G, Panero M, Duca S and Cauda F (2019). The homotopic connectivity of the functional brain: a meta-analytic approach. Scientific Reports, 9 (1).

[17] Niell CM and Stryker MP (2010). Modulation of visual responses by behavioral state in mouse visual cortex. Neuron, 65 (4), pp. 472-479.
[18] Passamonti L, Tsvetanov K, Jones P, Bevan-Jones W, Arnold R, Borchert R, Mak E, Su L, Obrien J and Rowe J (2019). Neuroinflammation and functional connectivity in Alzheimer's disease: Interactive influences on cognitive performance Journal of Neuroscience, 39 (36), pp. 7218-7226.

[19] Pesaran B, Pezaris JS, Sahani M, Mitra PP and Andersen RA (2002). Temporal structure in neuronal activity during working memory in macaque parietal cortex. Nature Neuroscience, 5 (8), pp. 805-811.

[20] Saba V, Premi E, Cristillo V, Gazzina S, Palluzzi F, Zanetti O, Gasparotti R, Padovani A, Borroni B and Grassi M (2019). Brain connectivity and informationflow breakdown revealed by a minimum spanning tree-based analysis of MRI data in behavioral variant frontotemporal dementia. Frontiers in Neuroscience, 13(211).

[21] Saleem AB, Lien AD, Krumin M, Haider B, Rosón MR, Ayaz A, Reinhold K, Busse L, Carandini M and Harris KD (2017). Subcortical source and modulation of the narrowband gamma oscillation in mouse visual cortex. Neuron, 93 (2), 315-322.

[22] Shovon M, Nandagopal N, Vijayalakshmi R, Du JT and Cocks B (2016). Directed connectivity analysis of functional brain networks during cognitive activity using transfer entropy. Neural Processing Letters., 45(3), pp. 807-824.

[23] Sohal VS, Zhang F, Yizhar O and Deisseroth K (2009). Parvalbumin neurons and gamma rhythms enhance cortical circuit performance. Nature, 459 (7247), pp. 698-702.

[24] Storchi R, Bedford RA, Martial FP, Allen AE, Wynne J, Montemurro MA, Petersen RS and Lucas RJ (2017). Modulation of fast narrowband oscillations in the mouse retina and dLGN according to background light intensity. Neuron, 93 (2), pp. 299-307.

[25] Towle VL, Hunter JD, Edgar JC, Chkhenkeli SA, Castelle MC, Frim DM, Kohrman M and Hecox KE (2007). Frequency domain analysis of human subdural recordings. Journal of Clinical Neurophysiology, 24 (2), pp. 205-213.

[26] Welle CG and Contreras D (2016). Sensory-driven and spontaneous gamma oscillations engage distinct cortical circuitry. Journal of Neurophysiology, 115 (4), pp. 1821-1835.

[27] Welle C and Contreras D (2017). New light on gamma oscillations. Neuron, 93 (2), pp. 247-249.

[28] Zhang T, Wu J, Li F, Caffo B and Boatman-Reich D (2015). A dynamic directional model for effective brain connectivity using electrocorticographic (ECoG) time series. Journal of the American Statistical Association, 110(509), pp. 93-106.

[29] Zhou S and Yu Y (2018). Synaptic E-I balance underlies efficient neural coding. Frontiers in Neuroscience, 12(46). 